\title{
Hemispheric symmetry in duration of visible persistence
}

\author{
VINCENT DI LOLLO \\ University of Alberta, Edmonton, Alberta, Canada T6G $2 E 9$
}

\begin{abstract}
Nine right-handed subjects performed a visual task requiring perceptual integration of a pattern whose parts were displayed sequentially in time. Correct performance on the task depended critically on the simultaneous visibility of all parts of the pattern; duration of visible persistence could therefore be gauged by varying the duration of the temporal interval between successive portions of the display. The pattern was displayed either foveally or parafoveally in either visual field. Analysis of overall performance and of distribution of errors at each temporal interval revealed more accurate performance for foveal displays but no hemispheric asymmetries in duration of visible persistence. These and other results reported in the literature are interpreted in terms of Moscovitch's (1979) information-processing model of hemispheric functioning.
\end{abstract}

Brief visual stimuli displayed for less than about $100 \mathrm{msec}$ tend to be perceived as being displayed for a somewhat longer duration (Di Lollo, 1977, 1980; Efron, 1973; Haber, 1971). The short-lived representation that remains available after the termination of the inducing stimulus has been known as "iconic memory" (Neisser, 1967) and has been extensively investigated since it was described systematically by Sperling (1960).

In an intriguing recent development, differences in perceived duration have been reported between the two cerebral hemispheres. Polzella, Da Polito, and Hinsman (1977) displayed random aggregates of dots to the left visual field (LVF) or to the right visual field (RVF) of subjects who were required to estimate the apparent duration of the display. Numerosity of dots in each display was varied between one and five; duration of each display was varied between 16 and $100 \mathrm{msec}$. Subjects expressed their judgments of the apparent duration of each display by placing a mark on a 5-point bipolar scale consisting of a line that had been marked off into five equal segments and had been labeled "shortest" at one end and "longest" at the other. The results showed that the judged duration of a display was greater when the dot pattern was shown in the RVF than when it was shown in the LVF. To the contrary, Erwin and Nebes (Note 1) found that perceived duration of briefly displayed alphabetic characters was greater in the left than in the right visual field. These diverging results could be attributed to interhemispheric differences in the processing of verbal and nonverbal materials. But can

\footnotetext{
This research was supported by Natural Sciences and Engineering Research Council of Canada Grant A6592. Requests for reprints should be sent to Vincent Di Lollo, Department of Psychology, University of Alberta, Edmonton, Alberta, Canada T6G 2E9. I wish to thank Alinda Friedman for her helpful comments.
}

the results be ascribed to interhemispheric differences in duration of visible persistence, as was suggested by Erwin and Nebes (Note 1)? To answer this question, let us examine Erwin and Nebes' experimental paradigm that yielded the strongest laterality effects: Sets of alphabetic characters were flashed to the LVF or to the RVF of subjects, who were required to report verbally the contents of the display. Two different reaction times (RT) were measured in separate sets of trials: RT to the onset of the display and RT to the termination of the display. Subtraction of mean onset $\mathrm{RT}$ from mean termination RT yielded the phenomenal duration of the display. In turn, duration of visible persistence was calculated by subtracting the actual duration of the physical display from the computed phenomenal duration. By this technique (known as subtractive reaction time technique), Erwin and Nebes found greater subtractive RTs to stimuli displayed in the LVF and inferred that the right cerebral hemisphere generated longer durations of visible persistence.

However, it is not entirely clear that the measure obtained by Erwin and Nebes (Note 1) was limited solely by the duration of visible persistence. Indeed, an alternative account of these results would be that the letters in the display might have remained visible for an equal amount of time in both hemispheres, but that unequal additional time was taken after the termination of visible persistence to decode and interpret the contents of the display. On this option, Erwin and Nebes' results would be attributable not to hemispheric differences in duration of visible persistence but to hemispheric asymmetries in the duration of higher processes taking place after the termination of visible persistence.

To clarify this argument, it is necessary to distinguish between visible and nonvisible forms of sensory persistence. Such separation is akin to Sperling's (1967) 
distinction between visible and nonvisible memory traces, and parallels the distinction made by Phillips (1974) and by Turvey (1978) between visible and schematic persistence. While the former is an immediate and short-lived visible image of the display, the latter is said to occur at further processing stages. Although arising from visual stimulation and still maintaining some structural information about the display, schematic persistence is held to be no longer visible. A compelling case for a twofold classification of visual persistence has been presented by Coltheart (1980) in his recent treatise on iconic memory.

Were the laterality differences reported by Erwin and Nebes (Note 1) and by Polzella et al. (1977) primarily based on visible persistence, on schematic persistence, or on both? Neither study can provide a pertinent answer because neither experimental task was selectively sensitive to one or the other form of persistence. Yet, the issue is clearly significant to the study of hemispheric functioning, its salience underscored by Moscovitch's (1979) claim that functional differences between the hemispheres become evident only at levels presumed to be beyond those characterized by visible persistence.

The present study was designed to examine laterality differences in duration of visible persistence. For this purpose, observers were engaged in a task requiring that specifically visible properties of the stimulus still be available after the termination of the external display. This was done by employing a task that required simultaneous visibility of a configuration whose parts were presented sequentially in time. The task, similar to that developed by Eriksen and Collins (1968), was first employed by Hogben and Di Lollo (1974). The stimulus configuration used in the task is a matrix of 16 dots arranged in four rows and columns, plotted on an oscilloscope. One of the dots, chosen randomly on each trial, is not plotted. The observer is required to report the location of the missing dot within the matrix. If the 15 dots are plotted singly and sequentially over a period not exceeding about $100 \mathrm{msec}$, all dots are seen clearly and simultaneously, with the empty matrix location easily detectable. But, if the plotting period exceeds about $120 \mathrm{msec}$ (i.e., if the temporal separation between successive dots is increased), the dots displayed early in the sequence appear to be missing and become easily confused with the unplotted dot. When this happens, the subject is confronted with several ostensibly empty matrix locations from which to guess, and accuracy of performance deteriorates rapidly and dramatically (Hogben \& Di Lollo, 1974). This task is notable for the prominent role played by visible persistence: Unless all parts of the serial display are simultaneously visible, successful performance is virtually impossible. Interhemispheric differences in duration of visible persistence may be gauged by displaying the matrix in either visual field.

\section{METHOD}

\section{Subjects}

Nine subjects, four males and five females, served in this experiment. All had normal or corrected-to-normal vision; all were right-handed with no familial history of sinistrality.

\section{Visual Display}

The display consisted of 15 of the 16 dots defining a 4 by 4 square matrix plotted on a gridless Hewlett-Packard 1333A pointplotter equipped with fast P15 phosphor. Displays were generated by a PDP-8/L computer, which also performed all the timing and scoring functions. Subjects sat inside a lightproof cubicle and viewed the display binocularly through a Tektronix Model 0160154-00 viewing hood. At the viewing distance of $28 \mathrm{~cm}$, the dot matrix subtended a visual angle of approximately $2.1 \mathrm{deg}$; the angular separation between adjacent dots in the matrix was approximately $.7 \mathrm{deg}$. Luminance of the displays was set at a comfortable viewing level, which was kept constant from day to day by the calibration procedure described by Di Lollo (1979).

A trial was initiated by the depression of a pushbutton, hand held by the subject. Upon a button press, 15 of the 16 matrix dots were displayed one at a time at regular intervals in a sequence of locations that varied randomly from trial to trial. The location of the unplotted dot also varied randomly on each trial. The subject's task was to identify the location of the missing dot by naming its coordinates within the matrix. Each dot was itensified for only $1 \mu \mathrm{sec}$ on the viewing surface. Successive dots in the plotting sequence were separated by regular interdot iniervals during which no dots were plotted on the screen. In describing the duration of a display, it is convenient to employ the term plotting interval, which refers to the total time that elapsed between the plotting of the first and the last dots in the display. For example, since each display consisted of 15 dots (14 interdot intervals), an interdot interval of $10 \mathrm{msec}$ yielded a total plotting interval of $140 \mathrm{msec}$, the actual plotting time for any one dot being negligible.

Two fixation points were employed: one located $2 \mathrm{deg}$ above and the other 2 deg below the center of the display surface. Subjects were instructed to fixate on an imaginary point halfway between the two fixation points.

\section{Design and Procedure}

Two independent variables were manipulated: duration of total plotting interval and spatial location of the display with respect to fixation. There were three plotting intervals: 50,100 , or $150 \mathrm{msec}$, corresponding to interdot intervals of approximately $3.57,7.14$, and $10.71 \mathrm{msec}$, respectively. As to spatial location, the matrix was displayed foveally, in the LVF, or in the RVF. For foveal displays, the matrix was centered on the imaginary fixation point described above. For LVF and RVF displays, the matrix was centered approximately $3.63 \mathrm{deg}$ to the left or to the right, respectively, of the imaginary fixation point.

The three levels of plotting interval and of spatial location were combined factorially to yield a total of nine experimental conditions. An experimental session consisted of 90 randomly ordered trials composed of 10 presentations of the dot matrix for each of the nine experimental conditions. The 90 trials occurred in a different random order for each session and were completed within $10 \mathrm{~min}$. Each subject participated in 10 separate sessions over a period of several days. This procedure yielded 100 trials per sutject for each of the nine experimental conditions. During each session, the subject sat in a lightproof cubicle and initiated each trial by a button press. After each trial, the subject spoke the response over a headphone-microphone set to the experimenter, who entered it into the computer. If the subject made an incorrect response, the computer printed out a code that identified the experimental condition and the ordinal position (within the plotting sequence on that particular trial) of the dot that had been erroneously named as missing. 


\section{RESULTS}

Percentage of errors made at each plotting interval at the three display locations (foveal, LVF, RVF) are shown in Figure 1, averaged over the nine subjects. In agreement with earlier findings (Di Lollo, 1977; Hogben \& Di Lollo, 1974), overall performance deteriorated markedly as the plotting interval was increased. An analysis of variance performed on these data revealed significant effects of plotting interval $[F(2,24)=27.72, p<.001]$ and of display location $[\mathrm{F}(2,24)=8.52, \mathrm{p}<.002]$, but no significant interaction effect $[F(4,72)<1]$. Pairwise comparisons showed that the significant effect of display location was due entirely to the differences between the foveal and the parafoveal displays (see Figure 1). Notably, no significant differences were found between the results for LVF and RVF, showing that, with this task, there is no evidence of functional cerebral differences with respect to duration of visible persistence.

A finer analysis of the incidence of errors is permitted by the present experimental paradigm. In performing the matrix task, an error occurred whenever a dot that had actually been plotted was named as missing. The scoring procedure kept track of the order in which the dots had been plotted on any given trial and, within that plotting sequence, it identified the ordinal position of the dot that had been named erroneously as missing. Figure 2 shows the results

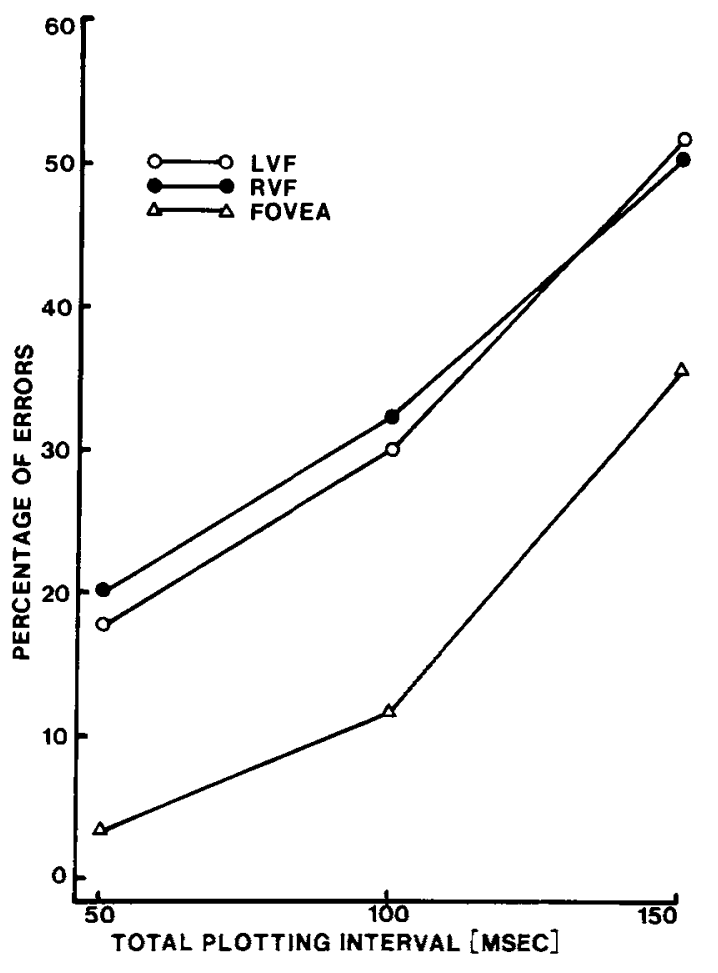

Figure 1. Mean percentage of errors at each plotting interval for stimuli displayed in the left visual field (LVF), in the right visual field (RVF), or foveally.


ORDINAL POSITION IN PLOTTING SEQUENCE

Figure 2. Temporal distribution of errors. Panels $\mathbf{A}, \mathrm{B}$, and $\mathbf{C}$ show error distributions for plotting intervals of 50,100 , and $150 \mathrm{msec}$, respectively. Within each panel, error distributions are shown for stimuli displayed to the left visual field (LVF), to the right visual field (RVF), or foveally. In each graph, the abscissa indicates the ordinal position of each dot within the plotting sequence, irrespective of spatial location; the ordinate shows the percentage of trials on which a dot plotted at the indicated ordinal position was incorrectly identified as missing. 
of this error analysis. Each curve in Figure 2 represents the average performance of the nine subjects, but is entirely representative of individual results. Panels A, $B$, and $C$ in Figure 2 show the results for the 50-, $100-$, and $150-\mathrm{msec}$ plotting intervals, respectively. Within each panel, the three curves show the results for each of the three locations (LVF, RVF, and fovea).

From Panel $\mathbf{A}$, it is clear that, at a plotting interval of $50 \mathrm{msec}$, the number of errors was extremely low and their distribution was uniform throughout the 15 ordinal positions in the plotting sequence: The most recent dots were just as likely to be confused with the empty matrix location as were dots plotted earlier in the sequence. This is hardly surprising since, at such brief plotting intervals, all dots in the display were seen clearly and simultaneously.

Levels of accuracy in LVF and RVF were practically identical, suggesting that, under conditions of virtual simultaneity of plotting, there were no hemispheric differences in the performance of this task. The more accurate performance obtained in the foveal displays was probably due to sharper acuity in foveal, rather than in parafoveal, areas.

Panels $B$ and $C$ in Figure 2 show that, as the duration of the total plotting interval was increased, there was an increasing tendency for dots that appeared early in the plotting sequence to be named as missing. This pattern of results is similar to that found in other studies (Di Lollo, 1977; Hogben \& Di Lollo, 1974) and suggests that visible persistence of the early dots had vanished by the time the last dot was plotted. The phenomenal appearance of the displays was in accordance with this suggestion: At the longer plotting intervals, the matrix was seen as having several empty locations from which to choose a response.

Of major interest to the present work is a comparison between the two visual hemifields. From Panels $B$ and $C$ in Figure 2, it is manifest that the temporal distribution of errors was essentially the same in LVF and RVF. Longer visible persistence in a given hemisphere might have been evidenced in Figure 2 by a reduced error rate at the early ordinal positions of displays shown to the contralateral retinal hemifield. For example, had duration of visible persistence been greater in, say, the right hemisphere, more of the early dots plotted in the LVF should have been still visible at the end of the display sequence. In turn, this would have caused the LVF curves (Panels B and C, Figure 2) to be lower than the RVF curves over some of the early ordinal positions in the plotting sequence. There is no hint of such differences in Figure 2. On the contrary, the error distributions shown in Figure 2 underscore the equivalence of the two hemispheres with respect to duration of visible persistence.

\section{DISCUSSION}

On the face of it, the experimental evidence appears to be highly discordant. It has been claimed that duration of visible persistence is greater in the right hemisphere (Erwin \& Nebes, Note 1), greater in the left hemisphere (Polzella et al., 1977), or equal in both hemispheres (the present work). In attempting to resolve these inconsistencies, it should be remembered that successful performance in the present study required an enduring visible representation of all dots in the display. On the other hand, the experimental tasks in the other studies could be performed without such visible representation. Put differently, while performance of the present task required visible persistence, the tasks in the other studies could be performed on the basis of schematic persistence. Bearing this in mind, it may be suggested that the divergent inferences regarding hemispheric asymmetries in visible persistence are in conflict with each other because they may be based on the duration of different processing events.

The ostensible inconsistencies can be resolved on the basis of information-processing theory. For this purpose, two assumptions are necessary. The first is the assumption traditionally made that the representation of a stimulus within the visual system consists of a neural code that undergoes a series of transformations as it proceeds through successive stages of processing. The second assumption is that visible persistence is to be regarded not as the decaying content of an iconic store (Neisser, 1967) but as the product of sensory coding activity at an early stage of visual information processing. In other words, visible persistence is assumed to arise over a brief interval corresponding to the duration of a very early processing stage. On this assumption, visible persistence endures during the time that the original stimulus is represented within the visual system by a given neural code and stops when the neural code is changed as processing continues to the next stage. Evidence in support of this hypothesis has been reviewed by Craik (1979) and by Di Lollo (1980).

Within the framework of information-processing theory, studies of hemispheric asymmetries have attempted to determine at what processing stage asymmetries first begin to emerge. This literature has been recently reviewed by Moscovitch (1979), who concluded that "hemispheric asymmetries emerge only at the level of a central processor that integrates information from the peripheral channels and represents it in terms of configural, relational, or categorical properties that reflect the mode of operation peculiar to the processor in each hemisphere"' (p. 388). Notably, the experimental evidence reviewed by Moscovitch showed that early stages of processing, concerned mainly with encoding physical features of the stimulus, are either common to, or function equivalently in, both hemispheres. On this theoretical perspective, the hemispheric asymmetries reported by Erwin and Nebes (Note 1) and by Polzella et al. (1977) are to be ascribed not to differences in duration of visible persistence 
but to hemispheric differences at higher levels of processing. This is clearly suggested by Erwin and Nebes' (Note 1) finding that hemispheric differences were obtained only when subjects were required to interpret and report (rather than merely observe) the verbal contents of the display. These activities probably involve higher levels of processing, which in turn are more likely to underlie schematic than visible persistence.

In contrast, in the present task, successful performance rests unambiguously upon visible persistence alone. Thus, from this perspective, the absence of hemispheric asymmetries in duration of visible persistence can be explained on the joint grounds that visible persistence is produced at an initial stage of processing, and that early stages of information processing are handled similarly and with equal efficiency by both hemispheres.

\section{REFERENCE NOTE}

1. Erwin, D. E., \& Nebes, R. D. Right hemispheric involvement in the functional properties of visual persistence. Paper presented at the Annual Meeting of the Eastern Psychological Association, New York City, April 21-24, 1976.

\section{REFERENCES}

Coltheart, M. Iconic memory and visible persistence. Perception \& Psychophysics, 1980, 27, 183-228.

CraIk, F. I. M. Human memory. In M. R. Rosenzweig \& L. W. Porter (Eds.), Annual Review of Psychology, 1979. 30. 63-102.

Di Lollo, V. Temporal characteristics of iconic memory. Nature, 1977, 267, 241-243.

Di Lollo, V. Luminous calibration of oscilloscopic displays.
Behavior Research Methods \& Instrumentation, 1979, 11, 419-421.

Di LoLLo, V. Temporal integration in visual memory. Journal of Experimental Psychology: General, 1980, 109, 75-97.

Efron, R. An invariant characteristic of perceptual systems in the time domain. In S. Kornblum (Ed.), Attention and performance IV. New York: Academic Press, 1973.

Erizsen, C. W.. \& Collins, J. F. Sensory traces versus the psychological moment in the temporal organization of form. Journal of Experimental Psychology, 1968, 77, 376-382.

HABER, R. N. Where are the visions in visual perception? In S. J. Segal (Ed.), The adaptive function of imagery. New York: Academic Press, 1971.

Hogben, J. H., \& Di Lollo. V. Perceptual integration and perceptual segregation of brief visual stimuli. Vision Research, $1974,14,1059-1069$.

Moscovitch, M. Information processing and the cerebral hemispheres. In M. S. Gazzaniga (Ed.), Handbook of behavioral neurobiology (Vol. 2). New York: Plenum Press, 1979.

Neısser, V. Cognitive psychology. New York: Appleton-CenturyCrofts, 1967.

Phillips, W. A. On the distinction between sensory storage and short-term visual memory. Perception \& Psychophysics, 1974, 16, 282-290.

Polzella, D. J.. Da Polito, F., \& Hinsman, M. C. Cerebral asymmetry in time perception. Perception \& Psychophysics, $1977,21,187-192$

SPERLing, G. The information available in brief visual presentations. Psychological Monographs, 1960, 74(Whole No. 498).

SPERling, G. Successive approximations to a model for shortterm memory. Acta Psychologica, 1967, 27, 285-292.

Turvey, M. T. Visual processing and short-term memory. In W. K. Estes (Ed.), Handbook of learning and cognitive processes (Vol. 5). Hillsdale, N.J: Erlbaum, 1978.

(Received for publication September 4, 1980; revision accepted October $10,1980$. 